\title{
The significance of measuring health related quality of life in type 2 diabetes mellitus
}

\author{
Paulomi M. Sudhir
}

Received: 6 May 2014 / Accepted: 13 May 2014 / Published online: 4 June 2014

(C) Research Society for Study of Diabetes in India 2014

The management of type 2 diabetes poses a significant challenge to both the treatment provider and the patient due to the complex behavioral and lifestyle changes that are required [1]. The importance of self care and metabolic control in delaying complications is also well recognized. While certain changes in lifestyle can indeed be beneficial and improve quality of life, others are likely to have a negative impact on the social and emotional functioning of the individual [2]. With newer medical advancements that delay complications and reduce mortality, the inclusion of quality of life as a component of health outcome has become crucial to the delivery of care.

Health related quality of life (HRQOL) is now recognized as being an inclusive term that assesses both self reported mental and physical functioning of an individual. HRQOL is defined as the "physical, psychological and social domains of health, seen as distinct areas that are influenced by a person's experiences, beliefs, expectations and perceptions" [3]. An important feature of this construct is that it acknowledges that an individual's personal views, judgments and preferences influence the perception of quality of life.

WHO Quality of Life- BREF, Medical Outcomes Study Short Forms (SF-12 and SF-36), the Sickness Impact Profile, and the Quality of Well-Being Scale are some examples of established generic measures of health related quality of life. The impact of a medical illness on overall psychological and emotional functioning can be captured by using disease specific measures. These measures assess the impact of disease specific worries, impact and overall satisfaction [4]. In a recent study, Nagpal, et al. (2010) describe the development of a 34 item quality of life measure developed for Indian patients [5].

P. M. Sudhir $(\bowtie)$

National Institute of Mental Health and Neurosciences, Bangalore, Karnataka, India

e-mail: paulomi.sudhir@gmail.com
In this issue, Patel et al. (2014) have addressed this important aspect of health outcome in patients with type 2 diabetes mellitus. They use both a generic as well as a specific measure for the assessment of psychological functioning. Their study taps essential factors that impact overall disease and patient management [6]. The appraisal or interpretation of having to experience a chronic illness and to live with it impacts the overall adjustment to the illness. The appraisal of illness management and self care plays an important role in both the actual self care and quality of life [7]. However this aspect is often ignored in the overall assessment of health outcome. A study on Indian patients with type 2 diabetes mellitus showed that patients who integrate themselves and emotionally adjust to diabetes, experience a greater sense of psychological wellbeing [8].

The Appraisal of Diabetes (ADS) detects individuals at risk for poor quality of life. As the name suggests it assesses how a person with diabetes assesses the illness and its impact, confidence in coping, worries about the future as well as uncertainty with regard to the future [9].

Patel et al. (2014) report that patients with poorer control reported greater negative appraisal of diabetes, suggesting that perhaps these patients not only experienced greater emotional distress over their overall control over diabetes, but also experienced significant worries and uncertainty [6]. Negative appraisals in terms of burden, uncertainty about the future, poor confidence over coping with the illness are likely to lead to lower quality of life and a greater sense of burden due to the illness. Female gender, greater complications and comorbidity are all likely to further increase this sense or burden and lower the sense of well-being as noted by Patel et al. and by previous studies $[2,8]$.

An interesting finding by the authors is that patients with better control over diabetes had lower quality of life pertaining to physical activities such as sleep, activity level and so on. This finding reiterates the point that 
biomedical outcomes and psychological outcomes such as quality of life or emotional adjustment must be measured separately, as better metabolic control need not always be associated with better quality of life. This also supports the view that the construct of health related quality of life is complex and multi-dimensional [10].

Understanding psychological factors such as appraisal and emotional functioning is all the more important as studies have shown that psychological problems adversely affect regimen adherence and eventually metabolic control [1]. Often health care providers do not feel confident in their ability to identify psychological problems in their patients or to provide the psychological support that patients are likely to need [11]. In addition there are several systemic issues such as inadequate number of diabetes educators. The DAWN 2 study (Diabetes Attitudes Wishes and Needs) conducted across 17 countries including India, points to the need for patient centered care, in which patient and family needs are addressed. With respect to India, nearly $52 \%$ reported diabetes related distress and $27 \%$ experienced diabetes related discrimination $[12,13]$.

Attempts to measure and understand these emotional and psychological influences on disease management, self care are important as they inform the clinician of more effective ways to manage the illness, and at the same time not increase the appraisal of burden by the patient.

Earlier studies from India, employing diabetes specific QOL questionnaires showed differences in living with diabetes $[14,15]$ and the ability of integrating themselves and adjusting to diabetes fared better [16]. Even though type 1 diabetes is less common in India, psychosocial support is an integrated component of management [17]. Rigorous evaluation of QOL requires language validated questionnaires (eg Singh, Bradley) [18], simple questions that enquire about the patient's experience of the illness, self care, psychological and emotional functioning can be included in routine care, thereby enhancing the quality of care. Assessment of biomedical outcomes in the absence of these psychological outcomes would ignore a very integral aspect of the patient's life.

\section{References}

1. Rubin RR. Facilitating self care in people with diabetes. Diabetes Spectr. 2001;14:55-7.

2. Rubin RR. Diabetes and quality of life. Diabetes Spectr. 2000;13:21.

3. Testa MA, Simonson DC. Assessment of quality-of-life outcomes. N Engl J Med. 1996;334:835-40.

4. Garratt AM, Schmidt L, Fitzpatrick R. Patient-assessed health outcome measures for diabetes: a structured review. Diabet Med. 2002;19:1-11.

5. Nagpal Kumar A, Kakar S, Bhartia A. The development of 'Quality of Life Instrument for Indian Diabetes Patients (QOLID): a validation and reliability study in middle and higher income groups. J Assoc Physicians India. 2010;58:295-304.

6. Patel B, Oza, B, Patel K, Malhotra S, Patel V. Health related quality of life in type-2 diabetic patients in Western India using World Health Organization Quality of Life-BREF and appraisal of diabetes scale. Intl J Diab Dev Countries. doi:10.1007/s13410-013-0162-y.

7. Watkins KW, Connell CM, Fitzgerald JT, Kliem L, Hickey L, Ingersoll-Dayton B. Effect of adults' self-regulation of diabetes on quality-of-life outcomes. Diabetes Care. 2000;23:1511-5.

8. Shobhana R, Rao RP, Lavanya A, Padma C, Vijay V, Ramachandran A. Quality of life and diabetes integration among subjects with type 2 diabetes. J Assoc Physicians India. 2003;51:363-5.

9. Carey MO, Jorgensen RS, Weinstock RP, Sprafkin LJ, Lantinga CLM, Carnrike Jr MT, et al. Reliability and validity of the appraisal of diabetes scale. J Behav Med. 1991;14:43-51.

10. Chatterji S, Ustün BL, Sadana R, Salomon JA, Mathers CD, Murray CJL. The conceptual basis for measuring and reporting on health Global Programme on Evidence for Health Policy Discussion Paper No. 45 World Health Organization 2002.

11. Huang ES, Brown SE, Ewigman BG, Foley EC, Meltzer DO. Patient perceptions of quality of life with diabetes-related complications and treatments. Diabetes Care. 2007;30:2478-83.

12. International Diabetes Federation. IDF Diabetes Atlas, 5th edn. Brussels, Belgium: International Diabetes Federation, 2011. http:// www.idf.org/diabetesatlas.

13. Kalra S, Holt R. DAWN2 study results: provision of quality team-based and individualized diabetes care. Diabetes Voice. 2013;58:29-31.

14. Sridhar GR, Madhu K. Psychosocial and cultural issues in diabetes mellitus. Current Sci. 2002;83:1556-64.

15. Sridhar GR, Madhu K, Veena S, Madhavi R, Sangeetha BS, Asha Rani. Living with diabetes: Indian experience. Diabetes \& Metabolic Syndrome: Clin Res Rev. 2007;1:181-187.

16. R Shobhana, P Rama Rao, A Lavanya, C Padma, V Vijay, A Ramachandran. Quality of Life and Diabetes Integration Among Subjects With Type 2 Diabetes. J Assoc Physicians India. 2003;51: 363-5.

17. Paulomi Matam Sudhir, V Kumaraiah, C Munichoodappa, KM Prasanna Kumar. Management of children with diabetes mellitus — psychosocial aspects. Intl J Diab Dev Countries. 2001;21:69-76.

18. Singh H, Bradley C. Quality of life in diabetes. Intl J Diab Dev Countries. 2006;26:7-10. 